\section{Clinical outcomes of intravenous levetiracetam treatment in patients with renal impairment}

\author{
Anyamanee Lapmag, 1,2 \\ Sunee Lertsinudom, $1-3$ \\ Aporanee Chaiyakam,1,2 \\ Kittisak Sawanyawisuth, 4 \\ Somsak Tiamkao, ${ }^{3,4}$ on behalf of \\ Integrated Epilepsy Research Group, \\ Khon Kaen University \\ 1Cinical Pharmacy Division, Faculty of \\ Pharmaceutical Sciences, Khon Kaen \\ University; ${ }^{2}$ The College of \\ Pharmacotherapy of Thailand, \\ Pharmacy Council, Bangkok; \\ 3Integrated Epilepsy Research Group, \\ Khon Kaen University; ${ }^{4}$ Department of \\ Medicine, Faculty of Medicine, Khon \\ Kaen University, Thailand
}

\section{Abstract \\ Intravenous levetiracetam has been} approved for use as an antiepileptic drug, as well as in cases of status epilepticus. There are few reports that detail the clinical data and outcomes associated with this antiepileptic drug, particularly in patients with renal impairment. This was a retrospective analytical study conducted at Khon Kaen University's Srinagarind Hospital in Thailand. The study period was between January 1, 2010 and December 31, 2014. The inclusion criteria were that patents were over 15 years old, had renal impairment, and had received intravenous levetiracetam treatment. The main clinical outcomes were seizure control and mortality. Clinical outcomes were compared between those with and without status epilepticus. Mortality of patients with status epilepticus were compared in terms of seizure control and order of intravenous levetiracetam treatment. During the study period, there were 247 patients who met the study criteria. The average age of the patients was 58 years with nearly equal sex distribution. Of those, 90 patients (36.4\%) had GRFs of less than $15 \mathrm{~mL} / \mathrm{min} / 1.73 \mathrm{~m}^{2}$ and 60 patients $(24.3 \%)$ received intravenous LEVE due to status epilepticus. The seizure control rates in the status epilepticus and non-status epilepticus groups were $36.7 \%$ and $88.7 \%$, respectively $(\mathrm{P}<0.001)$. The mortality rate did not differ significantly between the two groups $(33.3 \%$ vs $27.8 \% ; \mathrm{P}=0.418$ ). There was no significant overall difference in mortality rate between seizure-controlled and seizure-uncontrolled patients in the status epilepticus group. In the convulsive status epilepticus group, variations in terms of treatment order of intravenous levetiracetam and seizure control resulted in no significant difference in mortality rates $(\mathrm{P}=0.311)$. No major side effects were detected in any patients after the intravenous levetiracetam treatment. In conclusion, intravenous levetiracetam treatment was effective and safe in patients with renal impairment.

\section{Introduction}

Epilepsy is a common disease in clinical practice. There were at least 70 million people worldwide suffered from epilepsy in 2010. ${ }^{1}$ Untreated or uncontrolled epilepsy may lead to several serious conditions or complications including status epilepticus. The administration of antiepileptic drugs is the main method used in the treatment of epilepsy, and is aimed at to controlling seizures, avoiding side effects, and maintaining a good quality of life. ${ }^{2}$ Currently, there are at least 155 antiepileptic products registered in Hong Kong, including new antiepileptic drugs such as levetiracetam and zonisamide. ${ }^{3}$ The International League Against Epilepsy (ILAE) reported that further clinical studies are required to evaluate the relevant overall outcomes associated with antiepileptic drugs. ${ }^{4}$

Intravenous levetiracetam has been approved for use as an antiepileptic drug, as well as in cases of status epilepticus. 5 Although it is effective, the dosage for patients with renal impairment should be reduced. ${ }^{6}$ Since levetiracetam clearance via kidney is $66 \%$ in patients with renal impairment, its half-life may last for 25 hours. ${ }^{7}$ Additionally, body clearance decreases by $50 \%$ in patients with an estimated glomerular filtration rate of $30-50 \mathrm{~mL} / \mathrm{min}^{6}{ }^{6}$ Despite the widespread use of intravenous levetiracetam, there are few reports that detail the clinical data and outcomes associated with this antiepileptic drug, particularly in patients with renal impairment. This study aimed to evaluate the clinical use of intravenous levetiracetam in patients with renal impairment.

\section{Materials and Methods}

This was a retrospective analytical study conducted at Khon Kaen University's Srinagarind Hospital in Thailand. The study period was between January 1, 2010 and December 31, 2014. The inclusion criteria
Correspondence: Somsak Tiamkao, Department of Medicine, Faculty of Medicine, Khon Kaen University, 40002, Khon Kaen, Thailand.

E-mail: somtia@kku.ac.th

Key words: status epilepticus; dose; mortality

Contributions: the authors contributed equally

Conflict of interest: the authors declare no potential conflict of interest.

Funding: none.

Received for publication: 28 October 2017.

Revision received: 25 January 2018.

Accepted for publication: 9 March 2018.

This work is licensed under a Creative Commons Attribution NonCommercial 4.0 License (CC BY-NC 4.0).

(C) Copyright A. Lapmag et al., 2018

Licensee PAGEPress, Italy

Neurology International 2018; 10:7469

doi:10.4081/ni.2018.7469

were that patents were over 15 years old, had renal impairment, and had received intravenous levetiracetam treatment. The definition of renal impairment was based on creatinine clearance using the CockcroftGault formula adjusted by skin surface area. ${ }^{8}$ The study protocol was approved by the ethic committee in human research, Khon Kaen University (HE591031).

Medical records of all eligible patients were reviewed. Baseline characteristics, estimated glomerular filtration rate (GFR, $\mathrm{mL} / \mathrm{min}$ ), indications for intravenous levetiracetam treatment, details regarding intravenous levetiracetam treatment, and clinical outcomes were recorded. The main clinical outcomes were seizure control and mortality. The definitions for clinical terms were as follows: antiepileptic drug treatment order in cases of status epilepticus was determined after initial benzodiazepine treatment; seizure control indicated that seizures were under control and there were no recurrent seizures within 24 hours after treatment with intravenous levetiracetam; and death meant in-hospital mortality regardless of cause.

Statistical analysis. Data of all eligible patients were analyzed using descriptive statistics. Baseline clinical data and treatment with intravenous levetiracetam are presented as mean (SD) or number (percentage). Clinical outcomes were compared between those with and without status epilepticus using a Chi-square test, while mortality of patients with status epilepticus 
were compared in terms of seizure control and order of intravenous levetiracetam treatment using a Fisher's Exact test. Statistical significance was defined as a $\mathrm{P}$ value less than 0.05 . All statistical analysis was performed using STATA software version 10.1 (College Station, Texas, USA) and SPSS program version 16 (Chicago, Illinois, USA).

\section{Results}

During the study period, there were 247 patients who met the study criteria. The average age of the patients was 58 years with nearly equal sex distribution. Of those, 90 patients (36.4\%) had GRFs of less than $15 \mathrm{~mL} / \mathrm{min} / 1.73 \mathrm{~m}^{2}$ and 60 patients $(24.3 \%)$ received intravenous levetiracetam due to status epilepticus. Intravenous levetiracetam was administered as the first-line antiepileptic drug in 165 patients $(66.8 \%)$ and was given at a dose between 1-2 $\mathrm{gm} /$ day in 226 patients $(91.5 \%)$. There were 44 patients who received intravenous levetiracetam without dose adjustment for renal impairment, but $97.7 \%$ were given a dose that was within treatment dosage range (Table 1). The average expense per patient was 13,072 Baht (373.5 USD) which was due to the cost of intravenous levetiracetam, at 10,165 Baht (290.4 USD).

The seizure control rates in the status epilepticus and non-status epilepticus groups were $36.7 \%$ and $88.7 \%$, respectively $(\mathrm{P}<0.001)$. The mortality rate did not differ significantly between the two groups (33.3\% vs 27.8\%; P value 0.418). The most common cause of death in both groups was sepsis (44 patients) as shown in Table 2. There was no significant overall difference in mortality rate between seizure-controlled and seizure-uncontrolled patients in the status epilepticus group (Table 3). In the status epilepticus group, variations in terms of treatment order of intravenous levetiracetam and seizure control resulted in no sig-
Table 1. Baseline characteristics and treatment of patients with renal impairment who received intravenous levetiracetam $(n=247)$.

\begin{tabular}{lc}
\hline Factors & Values \\
Mean age (SD), years & $58.0(18.8)$ \\
Male sex & $120(48.6)$ \\
\hline Glomerular filtration rate (GFR), mL/min/l.73 m² & \\
$45-59$ & $63(25.5)$ \\
$30-44$ & $33(13.4)$ \\
$15-29$ & $61(24.7)$ \\
$<15$ & $90(36.4)$ \\
Indications & \\
Status epilepticus & $60(24.3)$ \\
Non-status epilepticus & $187(75.7)$ \\
Naïve to levetiracetam & $119(48.2)$ \\
Currently on levetiracetam & $19(7.7)$ \\
Pre-operative prophylaxis & $23(9.3)$ \\
Others & $26(10.5)$ \\
\hline Dose, mg/d & \\
500-999 & $17(6.9)$ \\
1000-1999 & $226(91.5)$ \\
2000-3000 & $4(1.6)$ \\
Inappropriate dose by GFR & $44(17.8)$ \\
Dose within therapeutic range & $43(97.7)$ \\
Treatment order & \\
First-line & $165(66.8)$ \\
Second-line & $64(25.9)$ \\
Third-line & $15(6.1)$ \\
Fourth-line & $3(1.2)$ \\
\hline Mean (SD) numbers of levetiracetam vials/patient & $25.2(24.6)$ \\
Mean (SD) of levetiracetam treatment, days & $8.6(11.3)$ \\
\hline
\end{tabular}

Table 2. Clinical outcomes of patients with renal impairment who received intravenous levetiracetam $(n=247)$ categorized by status epilepticus.

\begin{tabular}{lccc} 
Outcomes & Status epilepticus $(\mathrm{n}=60)$ Non- status epilepticus (n=187)P value \\
Seizure controlled & $22(36.7)$ & $166(88.7)$ & $<0.001$ \\
Death & $20(33.3)$ & $52(27.8)$ & 0.418 \\
Sepsis & 14 & 30 & \\
Gastric perforation/UGIB & 2 & 1 & \\
Severe metabolic disturbance & 2 & 7 & \\
Respiratory failure & 1 & 4 & \\
Sudden cardiac arrest/shock & 1 & 8 & \\
Hypovolemic shock & 0 & 1 & \\
Liver failure & 0 & 1 & \\
\hline
\end{tabular}

Data presented as number (percentage); UGBI: upper gastrointestinal bleeding.

Table 3. Mortality of status epilepticus patients with renal impairment who received intravenous levetiracetam (n=60) categorized by treatment order of intravenous levetiracetam and seizure control $(P=0.311)$.

\begin{tabular}{|c|c|c|c|}
\hline \multirow{2}{*}{ Order of intravenous levetiracetam } & \multicolumn{3}{|c|}{$\begin{array}{l}\text { Seizures uncontrolled } \\
\qquad(\mathrm{n}=38)\end{array}$} \\
\hline & Survived & Total & Died \\
\hline
\end{tabular}

\begin{tabular}{|c|c|c|c|c|c|c|c|}
\hline First line & 4 & 9 & 13 & 8 & 10 & 18 & 31 \\
\hline Second line & 4 & 1 & 5 & 3 & 10 & 13 & 18 \\
\hline Third line & 0 & 4 & 4 & 0 & 4 & 4 & 8 \\
\hline Fourth line & 0 & 0 & 0 & 1 & 2 & 3 & 3 \\
\hline Total & 8 & 14 & 22 & 12 & 26 & 38 & 60 \\
\hline
\end{tabular}


nificant difference in mortality rates, as shown in Table 3 (P value 0.311). No major side effects were detected in any patients after the intravenous levetiracetam treatment.

\section{Discussion and Conclusions}

Levetiracetam is a broad-spectrum antiepileptic drug and is approved as adjunctive therapy for focal-onset seizures, myoclonic seizure, juvenile myoclonic epilepsy, and primary generalized tonicclonic seizures in patients six years of age and older.9,10 The benefit of levetiracetam is its low drug interaction due to independent metabolism via the cytochrome P450 system. ${ }^{11}$ However, in patients with renal impairment, dose adjustment is required. ${ }^{12}$

In this study, the most common indication for intravenous levetiracetam treatment was non-status epilepticus (119 patients or $48.2 \%$ ), followed by status epilepticus ( 60 patients or $24.3 \%$ ). There were 23 patients (9.3\%) who received intravenous levetiracetam due to perioperative brain surgery prophylaxis. A previous study showed that intravenous levetiracetam reduced the rate of postoperative seizure in brain surgery from $15-20 \%$ to $7.3 \% .{ }^{13}$ Most patients received the appropriate dose for renal impairment (203 patients; 82.2\%). Although 44 patients received an inappropriate dose, the drug levels were within therapeutic range for 43 patients (97.7\%), as shown in Table 1. These findings may imply that intravenous levetiracetam may have a rather wide therapeutic range in renal impairment. Intravenous levetiracetam was prescribed as the first-line treatment at the highest ratio $(66.8 \%)$ due to low drug interaction. ${ }^{11}$

Intravenous levetiracetam was more effective in terms of seizure control in the non-status epilepticus group than in the status epilepticus group (88.7\% vs $36.7 \%)$. In this study, intravenous levetiracetam had a lower seizure-control rate than it did in a previous study. ${ }^{14}$ A study conducted by Oman found that intravenous levetiracetam had a seizure-control rate of $82 \%$ in 22 status epilepticus patients. In our previous

Table 4. Doses of intravenous levetiracetam renal impairment.

\begin{tabular}{lcc}
$\begin{array}{l}\text { Glomerular filtration rate } \\
\left(\mathrm{mL} / \mathrm{min} / 1.73 \mathrm{~m}^{2}\right)\end{array}$ & Total doses $(\mathrm{mg}) /$ day \\
$50-80$ & $500-1,000 \mathrm{q} 12 \mathrm{~h}$ & $1000-2000$ \\
$30-50$ & $250-750 \mathrm{mg} \mathrm{q} 12 \mathrm{~h}$ & $500-1500$ \\
\hline$<30$ & $250-500 \mathrm{mg} \mathrm{q} 12 \mathrm{~h}$ & $500-1000$ \\
\hline
\end{tabular}

study, the seizure-control rate of intravenous levetiracetamin cases of status epilepticus was lower than that of sodium valproate $(47.06 \%)$ but higher than phenytoin $(21.62 \%) .15$ These findings may be explained by differences in study population. Both previous studies were conducted in normal adults, but this study was performed in patients with renal impairment. This may indicate that intravenous levetiracetam may have lower efficacy in this setting. Note that mortality rates did not differ between status epilepticus and non-status epilepticus patients.

The overall mortality rate for the 60 patients with status epilepticus in the seizures-controlled group did not differ from that of the seizures-uncontrolled group (36.4\% vs $31.6 \%)$, as shown in Table 3 . Additionally, the order of intravenous levetiracetam did not affect the overall mortality rate ( $\mathrm{P}$ value 0.311 ). As previously reported, factors associated with mortality in status epilepticus are varied, but the types of antiepilptic drugs administered is not among them. ${ }^{15-18}$ The mortality rates in status epilepticus patients treated with phenytoin and sodium valproate were $29.73 \%$ and $11.76 \%$, respectively ( $\mathrm{P}$ value 0.189 ). ${ }^{15}$ Older age or early treatment may be associated with status epilepticus mortality. ${ }^{16-18}$ the results of this study in terms of order of intravenous levetiracetam treatment on mortality in status epilepticus patients.

There are some limitations to this study. First, mortality in this study was not specifically due to seizure and was recorded as inhospital mortality. No long-term mortality rates were recorded. Additionally, definition of seizure control in this study implied only 24 hours after seizure cessation. Second, there was no correlation data with regard to the level of renal impairment and treatment outcomes. Third, some data were missing due to the retrospective nature of the medical record reviews. Most patients in this study $(91.5 \%)$ received intravenous levetiracetam treatment between 1000-1999 $\mathrm{mg}$ /day despite almost equal of CKD level distribution (Table 1). These findings occurred because the recommended dose for those with GFR less than 50 is between Further studies may be needed to confirm
500-1500 mg/day (Table 4). ${ }^{19}$ Finally, as mentioned earlier, predictors for mortality and seizure control were not studied.

In conclusion, intravenous levetiracetam treatment was effective in patients with renal impairment.

\section{References}

1. Ngugi AK, Bottomley C, Kleinschmidt I, et al. Estimation of the burden of active and life-time epilepsy: a metaanalytic approach. Epilepsia 2010;51:883-90.

2. Schachter SC. Advances in the assessment of refractory epilepsy. Epilepsia 1993;34:S24-30.

3. Fong JK, Chan EL, Leung H, et al. An update of the Hong Kong Epilepsy Guideline: consensus statement on the use of antiepileptic drugs in Hong Kong. Hong Kong Med J 2017;23:74-88.

4. Glauser T, Ben-Menachem E, Bourgeois B, et al. Updated ILAE evidence review of antiepileptic drug efficacy and effectiveness as initial monotherapy for epileptic seizures and syndromes. Epilepsia 2013;54:551-63.

5. Trinka E, Dobesberger J. New treatment options in status epilepticus: a critical review on intravenous levetiracetam. Ther Adv Neurol Disord 2009;2:79-91.

6. Radtke RA. Pharmacokinetics of levetiracetam. Epilepsia 2001;42:24-71.

7. Wright C, Downing J, Mungall D, et al. Clinical pharmacology and pharmacokinetics of levetiracetam. Front Neurol 2013;4:192.

8. [No authors listed]. Chapter 1: Definition and classification of CKD. Kidney Int Suppl 2013;3:19-62.

9. Delanty N, Jones J, Tonner F. Adjunctive levetiracetam in children, adolescents, and adults with primary generalized seizures: open-label, noncomparative, multicenter, long-term follow-up study. Epilepsia 2012;53: 111-9.

10. Mbizvo GK, Dixon P, Hutton JL, Marson AG. Levetiracetam add-on for drug-resistant focal epilepsy: an updated Cochrane Review. Cochrane Database Syst Rev 2012;9:CD001901.

11. Otoul C, De Smedt H, Stockis A. Lack of pharmacokinetic interaction of levetiracetam on carbamazepine, valproic acid, topiramate, and lamotrigine in children with epilepsy. Epilepsia 2007;48:2111-5.

12. Yamamoto J, Toublanc N, Kumagai Y, Stockis A. Levetiracetam pharmacokinetics in Japanese subjects with renal 
impairment. Clin Drug Invest 15. Tiamkao S, Sawanyawisuth K, 2014:34:819-28.

13. Gokhale S, Khan SA, Agrawal A, Friedman AH, McDonagh DL. Levetiracetam seizure prophylaxis in craniotomy patients at high risk for postoperative seizures. Asian J Neurosurg 2013;8:169-73.

14. Gujjar AR, Nandhagopal R, Jacob PC, et al. Intravenous levetiracetam vs phenytoin for status epilepticus and cluster seizures: A prospective, randomized study. Seizure 2017;49:8-12.
Chancharoen A. The efficacy of intravenous sodium valproate and phenytoin as the first-line treatment in status epilepticus: a comparison study. BMC Neurol 2013;13:98.

16. Kantanen AM, Reinikainen M, Parviainen I, Kälviäinen R. Long-term outcome of refractory status epilepticus in adults: A retrospective population-based study. Epilepsy Res 2017;133:13-21.

17. Tiamkao S, Pranboon S,
Thepsuthammarat K, Sawanyawisuth K. Status epilepticus in the elderly patients: A national data study in Thailand. J Neurol Sci 2017;372:501-5. 18. Moghaddasi M, Joodat R, Ataei E. Evaluation of Shortterm Mortality of Status Epilepticus and Its Risk Factors. J Epilepsy Res 2015;5:13-6.

19. French J. Use of levetiracetam in special populations. Epilepsia 2001;42:403. 